\title{
orrespondence
}

\section{Rapid emergence of third generation cephalosporin resistant Shigella spp. in Southern Vietnam}

Diarrhoeal disease caused by third generation cephalosporin resistant Shigella spp. has been described in the past, but it remains a relatively infrequent disease. Here, we report 11 cases of childhood shigellosis caused by ceftriaxone-resistant organisms isolated in Southern Vietnam between May 2007 and January 2008. We predict that the emergence of such strains may become more frequent and will hamper effective treatment. Improved microbiological surveillance and clinical investigations are required for an accurate assessment of the problem.

There are an estimated 165 million shigellosis episodes annually worldwide, of which $64 \%$ of the patient burden is in children under the age of 5 years (Kotloff et al., 1999). Transmission of Shigella follows the faecal-oral route; hence, Shigella infections are commonly associated with poor sanitation and limited access to clean water. Antimicrobial therapy of Shigella infections hastens the clinical recovery, prevents complications and stops the dissemination of the bacteria back into the community. The emergence of multiple-drug resistant (MDR) strains of Shigella spp. over the last two decades clearly highlights the problem of MDR pathogenic enteric bacteria and makes the selection of treatment for shigellosis more problematical. The World Health Organization (WHO) currently recommends ciprofloxacin (or other fluoroquinolones) as the drug of choice for the therapy of Shigella infections in both adults and children. In addition, ceftriaxone, pivmecillinam (amdinocillin pivoxil) and azithromycin are considered as alternative drugs suitable for Shigella treatment (WHO, 2005). However, similar to other pathogenic enteric bacteria like Salmonella (Chau et al., 2007), strains of Shigella spp. that are resistant to ciprofloxacin have been described in Asia (Von Seidlein et al., 2006). Additionally, strains of Shigella spp. that produce extended spectrum $\beta$-lactamases (ESBL), conferring resistance to third generation cephalosporins have also been reported (Fortineau et al., 2001).

In Vietnam, MDR strains of Shigella spp. have been reported (Isenbarger et al., 2002; Vinh et al., 2000) but shigellosis caused by Shigella spp. that are resistant to third generation cephalosporins was, previously, unknown. During the course of a clinical shigellosis treatment trial conducted in Southern Vietnam from June 2006, we have identified several children infected with ceftriaxone-resistant Shigella. Here, we report the clinical, epidemiological and microbiological aspects of shigellosis in these patients.

The study was carried out at two locations in Southern Vietnam between June 2006 and January 2008. These locations were the Hospital for Tropical Diseases, Ho Chi Minh City, and Dong Thap Provincial Hospital, Dong Thap Province, in the Mekong Delta, approximately $180 \mathrm{~km}$ from Ho Chi Minh City. The criteria for enrolment in the clinical trial was: the patients had to be children under 15 years of age, of either sex, who were hospitalized because of acute dysentery (this was defined as passing bloody diarrhoea or mucoid stools with additional abdominal pain or tenesmus). Only those patients with written consent, given by parents or a guardian, were eligible to take part in the study. The clinical details were recorded in a study form.

Stool samples were taken from the patients on arrival in hospital, prior to the first administration of antimicrobials, then daily for the next 3 days, and additionally on a follow-up visit 1 week after being discharged from hospital. The stool samples were cultured by standard procedures in order to isolate Shigella spp. Antibiotic susceptibility testing was carried out using the disc diffusion method, according to Clinical and Laboratory Standards Institute (CLSI) guidelines. The MIC was measured using Etest strips (AB
Biodisk). The susceptibility of Shigella strains to ceftriaxone was used to ascertain strains that could generate ESBLs. Those strains that were identified as resistant to ceftriaxone using the disc diffusion susceptibility test were further subjected to the 'combination disc method' to confirm ESBL production. The combination disc method utilized discs containing cefotaxime $(30 \mu \mathrm{g})$ alone, ceftazidime $(30 \mu \mathrm{g})$ alone, and either antimicrobial combined with clavulanic acid $(10 \mu \mathrm{g})$. All antimicrobial testing was performed on Mueller-Hinton agar according to CLSI guidelines (CLSI, 2007).

The clinical study was initiated in June 2006; in the period from June 2006 to January 2008 we isolated 72 Shigella strains in total, of which $11(15.3 \%)$, were ceftriaxone resistant. These strains were isolated on admission from the stools of 11 children ( 7 boys, 4 girls) aged between 17 and 48 months. Eight of the patients came from Ho Chi Minh City, one came from Long An (near Ho Chi Minh City) and two from Dong Thap Province in the Mekong delta. The first ESBL-producing ceftriaxone-resistant Shigella sonnei strain was isolated from a faecal sample taken from a child from Ho Chi Minh City, who had severe dysentery, in May 2007. We then identified new ceftriaxone-resistant strains at a rate of one per month, until January 2008, when we isolated three strains. S. sonnei accounted for the majority of the ceftriaxone-resistant strains isolated (10/11). All of the S. sonnei were confirmed to produce ESBL due to an increase of $\geqslant 5 \mathrm{~mm}$ in zone diameter around cefotaxime/clavulanic acid disc compared to the zone around the cefotaxime disc, using the combination disc test. Shigella flexneri accounted for the remaining ceftriaxone-resistant strain (1/11). The S. flexneri isolate was found not to be ESBL producing as it only exhibited limited zone clearance with the combination disc test. This observation was corroborated by the lack of a 
characteristic bell-shaped zone of clearance between the two discs when the strain was grown on plates containing ceftriaxone and amoxicillin/clavulanic acid discs. All patients returned 1 week after discharge for a follow-up examination and were in good health. Faecal samples from 10 children were taken on the follow-up visit, all tested culture negative for Shigella.

Additionally, we retrospectively analysed a collection of 114 Shigella strains isolated between 2000 and 2002 for ceftriaxone resistance. We identified one strain of $S$. sonnei that displayed ESBL-mediated ceftriaxone resistance. This strain was isolated from a 10-month-old boy treated for acute watery diarrhoea in the Hospital for Tropical Diseases in February 2001. This, potentially, is the first recorded strain of third generation cephalosporin resistant S. sonnei in Vietnam.

Antimicrobial resistance is a well described phenomenon in the genus Shigella, but diarrhoeal disease caused by third generation cephalosporin resistant Shigella spp. is still uncommon. The first occurrence of third generation cephalosporin resistant $S$. flexneri was from the stool sample of a 16-month-old Algerian child hospitalized in Paris, France, in 1995; the microbiological details of this strain were published in 2001 (Fortineau et al., 2001). Since that time many strains of Shigella spp. harbouring different types of ESBLs have been reported in developed and developing countries in Asia (Cheung et al., 2005; Chuang et al., 2006; Kim et al., 2004; Pai et al., 2001; Rahman et al., 2004; Xiong et al., 2007). However, to the best of our knowledge this is the first report of ESBLproducing Shigella spp. in Vietnam.

The clinical patterns of these cases were typical of dysentery caused by a Shigella infection. Despite 9/10 S. sonnei strains in this series demonstrating co-resistance to nalidixic acid, all patients responded to oral fluoroquinolones (ciprofloxacin, gatifloxacin or norfloxacin). None of the patients had any clinical signs or symptoms of Shigella infection within 3 days of the onset of treatment. Additionally, all faecal samples from the patients taken after treatment and on follow-up visits were negative for Shigella. These data show that resistance to third generation cephalosporins did not prolong the shedding of Shigella provided the appropriate antimicrobials were administered. The molecular characteristics of the ESBL-encoding genes harboured by the $S$. sonnei strains in these isolates are currently being investigated and will be reported elsewhere. However, preliminary data suggest that ceftriaxone resistance in these strains is mediated by a plasmid-borne CTX-M class ESBLencoding gene. The molecular mechanism of the ESBL negative, ceftriaxone-resistant $S$. flexneri is also under investigation.

Our observations demonstrate that emergence of ceftriaxone-resistant shigellae in Southern Vietnam has not occurred by chance and the ability of these strains to produce ESBLs appears to be evolutionarily advantageous. The simple availability of antibiotics in Vietnam predicts that drug resistance in the community is rife. It is also known that ESBL-encoding genes can be carried on plasmids by non-pathogenic, commensal organisms, suggesting that spread of resistance genes to pathogenic bacteria may occur at a significant rate. The genetic transfer of drug resistance genes may not be of immediate concern for the treating clinicians, but will pose a potential problem in the future. We predict that the isolation of ceftriaxone-resistant shigellae and other Enterobacteriaceae will become increasingly common in Vietnam in the coming years. Currently, we have effective optional therapies; however, ceftriaxone is used in the hospital here in Vietnam as an alternative when the patient does not respond to fluoroquinolone treatment. A marked increase in resistance to nalidixic acid and newer fluoroquinolones, combined with ESBL-mediated third generation cephalosporin resistance would leave limited treatment options for those with life-threatening bacterial infections.

The majority of studies published in recent years on ESBL-producing Shigella have described mainly the transfer mechanisms and the molecular types of the $\beta$ -

lactamases produced. In order to have a comprehensive perspective on the clinical manifestations and outcomes of the illness caused by ESBL-producing shigellae more patient-oriented case series reports are required. Moreover, ESBL-producing strains of Shigella may be overlooked if screening for ESBLs is not routine in diagnostic microbiology laboratories. Therefore, more vigilance is required in detecting ESBL-producing Shigella strains and a worldwide rapid reporting system will allow the monitoring of the dissemination of such strains and whether diarrhoeal diseases caused by third generation cephalosporin resistant Shigella is an emerging trend worldwide.

\section{Ha Vinh, ${ }^{1,2}$ Stephen Baker, ${ }^{1}$ James Campbell, ${ }^{1}$ Nguyen Van Minh Hoang, ${ }^{1}$ Ha Thi Loan, ${ }^{2}$ Mai Thu Chinh, ${ }^{2}$ Vo Thi Cuc Anh, ${ }^{3}$ To Song Diep, ${ }^{2}$ Le Thi Phuong, ${ }^{3}$ Constance Schultsz ${ }^{1}$ and Jeremy Farrar ${ }^{1}$}

\section{${ }^{1}$ Oxford University Clinical Research Unit, Hospital for Tropical Diseases, Ho Chi Minh City, Vietnam \\ ${ }^{2}$ Hospital for Tropical Diseases, Ho Chi Minh City, Vietnam \\ ${ }^{3}$ Dong Thap Provincial Hospital, Dong Thap Province, Vietnam}

Correspondence: Stephen Baker (sbaker@oucru.org)

Chau, T. T., Campbell, J. I., Galindo, C. M., Van Minh Hoang, N., Diep, T. S., Nga, T. T., Van Vinh Chau, N., Tuan, P. Q., Page, A. L. \& other authors (2007). Antimicrobial drug resistance of Salmonella enterica serovar Typhi in Asia and molecular mechanism of reduced susceptibility to the fluoroquinolones. Antimicrob Agents Chemother 51, 4315-4323.

Cheung, T. K., Chu, Y. W., Tsang, G. K., Ngan, J. Y., Hui, I. S. \& Kam, K. M. (2005). Emergence of CTXM-type $\beta$-lactam resistance in Shigella spp. in Hong Kong. Int J Antimicrob Agents 25, 350-352.

Chuang, Y. Y., Huang, Y. C. \& Lin, S. Y. (2006). Outbreak of Shigella sonnei gastroenteritis in northeastern Taiwan. Pediatr Infect Dis J 25, 92-94.

CLSI (2007). Performance Standards for Antimicrobial Susceptibility Testing, seventeenth informational supplement, 27(1). Wayne, PA: Clinical and Laboratory Standards Institute.

Fortineau, N., Naas, T., Gaillot, O. \& Nordmann, P. (2001). SHV-type extended-spectrum $\beta$-lactamase in a Shigella flexneri clinical isolate. J Antimicrob Chemother 47, 685-688.

Isenbarger, D. W., Hoge, C. W., Srijan, A., Pitarangsi, C., Vithayasai, N., Bodhidatta, L., Hickey, K. W. \& Cam, P. D. (2002). Comparative antibiotic resistance of diarrheal pathogens from Vietnam and Thailand, 1996-1999. Emerg Infect Dis 8, 175-180. 
Kim, S., Kim, J., Kang, Y., Park, Y. \& Lee, B. (2004). Occurrence of extended-spectrum $\beta$ lactamases in members of the genus Shigella in the Republic of Korea. J Clin Microbiol 42, 52645269 .

Kotloff, K. L., Winickoff, J. P., Ivanoff, B., Clemens, J. D., Swerdlow, D. L., Sansonetti, P. J., Adak, G. K. \& Levine, M. M. (1999). Global burden of Shigella infections: implications for vaccine development and implementation of control strategies. Bull World Health Organ 77, 651-666.

Pai, H., Choi, E. H., Lee, H. J., Hong, J. Y. \& Jacoby, G. A. (2001). Identification of CTX-M14 extended-spectrum $\beta$-lactamase in clinical isolates of Shigella sonnei, Escherichia coli, and Klebsiella pneumoniae in Korea. J Clin Microbiol 39, 3747-3749.

Rahman, M., Shoma, S., Rashid, H., Siddique, A. K., Nair, G. B. \& Sack, D. A. (2004). Extendedspectrum $\beta$-lactamase-mediated third-

generation cephalosporin resistance in Shigella isolates in Bangladesh. J Antimicrob Chemother 54, 846-847.

Vinh, H., Wain, J., Chinh, M. T., Tam, C. T., Trang, P. T., Nga, D., Echeverria, P., Diep, T. S., White, N. J. \& Parry, C. M. (2000). Treatment of bacillary dysentery in Vietnamese children: two doses of ofloxacin versus 5-days nalidixic acid. Trans R Soc Trop Med Hyg 94, 323-326.
Von Seidlein, L., Kim, D. R., Ali, M., Lee, H., Wang, X., Thiem, V. D., Canh Do, G.,

Chaicumpa, W., Agtini, M. D. \& other authors (2006). A multicentre study of Shigella diarrhoea in six Asian countries: disease burden, clinical manifestations, and microbiology. PLoS Med 3, e353.

WHO (2005). Guidelines for the Control of Shigellosis, Including Epidemics due to Shigella dysenteriae type 1. Geneva: World Health Organization

Xiong, Z., Li, T., Xu, Y. \& Li, J. (2007). Detection of CTX-M-14 extended-spectrum $\beta$-lactamase in Shigella sonnei isolates from China. J Infect $\mathbf{5 5}$, e125-e128. 